\title{
Accelerating Mean Shift Segmentation Algorithm on Hybrid CPU/GPU Platforms
}

\author{
Liang Men, Miaoqing Huang, John Gauch \\ Department of Computer Science and Computer Engineering \\ University of Arkansas \\ $\{$ mliang,mqhuang,jgauch\}@uark.edu
}

\begin{abstract}
Image segmentation is a very important step in many GIS applications. Mean shift is an advanced and versatile technique for clusteringbased segmentation, and is favored in many cases because it is nonparametric. However, mean shift is very computationally intensive compared with other simple methods such as $k$-means. In this work, we present a hybrid design of mean shift algorithm on a computer platform consisting of both CPUs and GPUs. By taking advantages of the massive parallelism and the advanced memory hierarchy on Nvidia's Fermi GPU, the hybrid design achieves a $20 \times$ speedup compared with the pure CPU implementation when dealing with images bigger than $1024 \times 1024$ pixels.
\end{abstract}

Keywords: Image segmentation; mean shift, $k$-means clustering, GPU.

\section{Introduction}

Segmentation is the process of partitioning a digital image into multiple segments to simplify and/or change the representation of an image into a fashion that is more meaningful and easier to analyze. Image segmentation is typically used to locate objects and boundaries (lines, curves, etc.) in images [7]. The result of image segmentation is a set of segments that collectively cover the entire image, or a set of contours extracted from the image. Many methods have been designed to achieve the image segmentation goal, such as thresholding, clustering, edge detection, region growing among others.

In this work, we focus on accelerating the mean shift image segmentation algorithm on hybrid CPU/GPU computer platforms. Mean shift was introduced in Fukunaga and Hostetler [4] and has been extended to be applicable in other fields like Computer Vision [1,3]. Mean shift is a versatile non-parametric iterative algorithm that has better robustness than other clustering-based image segmentation algorithms such as $k$-means [5]. However, mean shift algorithm is computationally intensive. It's time complexity is $O\left(T n^{2}\right)$, where $T$ is the number of iterations for processing each data point and $n$ is the total number of data points in the data set, e.g., the number of pixels in the image. As it is shown in Section 4, the processing time of a large image becomes prohibitively long. This 
prompts the use of graphics processing units to accelerate the most computationally intensive part of the mean shift algorithm. Although the processing of an individual pixel is an iterative process, which is suitable to execute on CPU, the processing of individual pixels is independent, which provides a good chance to improve the performance by concurrently processing hundreds of pixels on massively parallel GPUs.

The remainder of the text is organized as follows. In Section 2 the mean shift algorithm is briefly introduced. The implementation detail is described in Section 3. Section 4 presents the results, and the conclusions are given in Section 5 .

\section{Mean Shift Algorithm}

Mean shift considers feature space as an empirical probability density function. If the input is a set of points then mean shift considers them as sampled from the underlying probability density function. If dense regions are present in the feature space, then they correspond to the local maxima of the probability density function. For each data point, mean shift associates it with the nearby peak of the data set's probability density function. For each data point, mean shift defines a window around it and computes the mean of the data point. Then it shifts the center of the window to the mean and repeats the algorithm till it converges. After each iteration, we can consider that the window shifts to a more denser region of the data set. At the high level, we can specify mean shift as follows:

1. Define a window around each data point;

2. Compute the mean of data within the window;

3. Shift the center of the window to the mean and repeat till convergence, i.e., the center of the window no longer shifts.

This process is illustrated in Fig. 1 in which particles are used as an example. The original position of the window is shown in Fig. 1(a). The geometric center of the window (i.e., $G C_{1}$ ) does not overlap with the center of the mass (i.e., $\left.M C_{1}\right)$. Therefore, the geometric center of the window is shifted to the center of the mass (i.e., $G C_{2} \leftarrow M C_{1}$ ) in Fig. 1(b). Then a new center of the mass is calculated (i.e., $M C_{2}$ ). If the geometric center and the mass center do not overlap, the geometric center will keep shifting until these two centers overlap (i.e., $G C_{n}=M C_{n}$ ), as shown in Fig. $1(\mathrm{~d})$.

In image segmentation, each pixel is dealt with as a data point. The coordinate of the pixel is initially set as the geometric center of the window. Then a center of pixel intensity is calculated using the pixels within the window. The geometric center of the window will shift to intensify center of the window until these two centers overlap, i.e., the convergence is reached. 


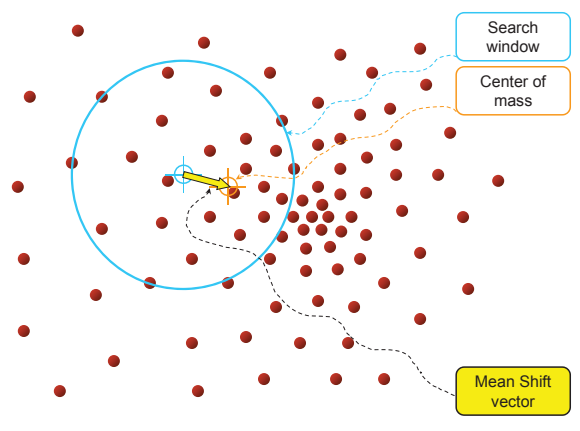

(a) Step 1 .

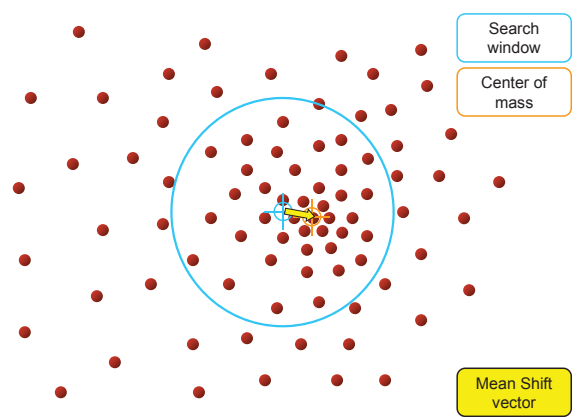

(c) Step 3 .

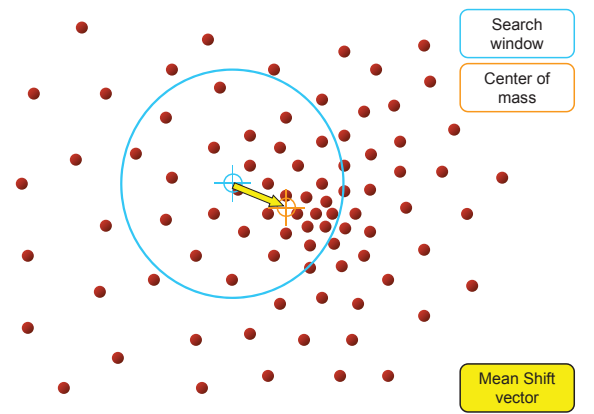

(b) Step 2.

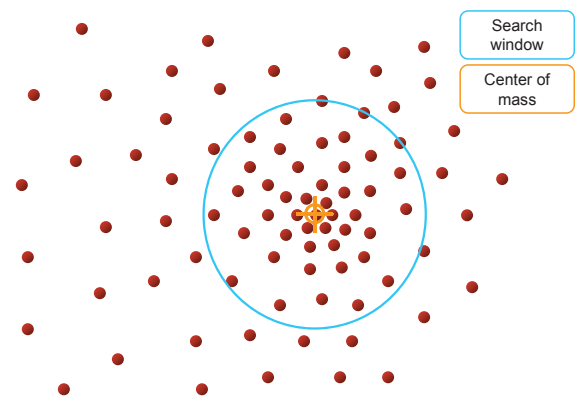

(d) Step 4: converge.

Fig. 1. The steps in mean shift algorithm using mass as an example.

\section{Hybrid Design of Mean Shift Image Segmentation}

The pure software implementation of the mean shift algorithm is adapted from the open source code developed by the Robust Image Understanding Lab at Rutgers University and is based on papers [2,3]. Given an image, the segmentation consists of two major steps. In the first step, the original image is filtered using mean shift method. Two parameters are needed, the spatial bandwidth $\left(h_{s}\right)$ and the range bandwidth $\left(h_{r}\right)$. The $h_{s}$ defines the radius of the window used in mean shift. The filtered image consists of numerous regions. In the second step, i.e., region fusion, three sub-steps are carried out in sequence, including connecting regions, applying transitive closure, and pruning spurious regions.

In our experiments, it is found that the first step, i.e., mean shift filtering, takes significantly longer time than the second step when the image size is smaller 


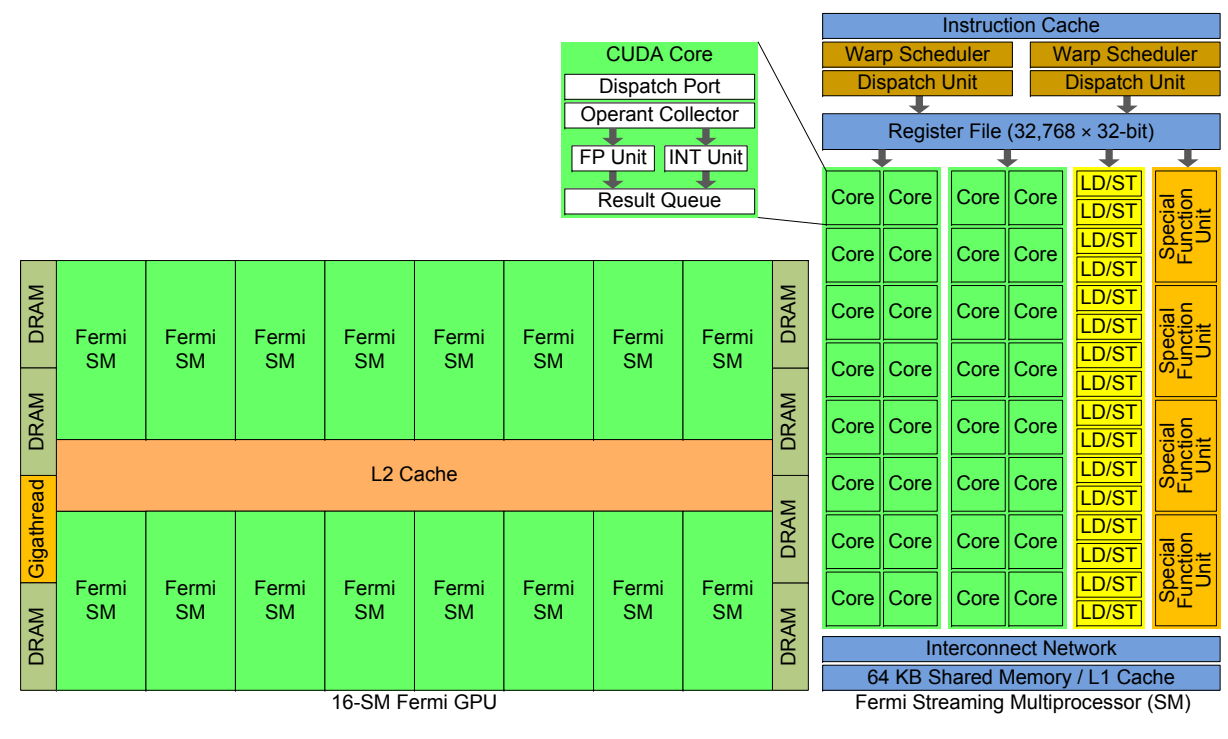

Fig. 2. Nvidia's Fermi GPU Architecture.

than $2,048 \times 2,048$, therefore the mean shift filtering step is executed on GPU and the region fusion step runs on CPU.

Given a pixel $P_{a}$ in the source image and a search window with radius $h_{s}$, the mean shift process is to repeatedly calculate the mean shift vector $\overrightarrow{M h}$ (shown in Fig. 1) until the squared magnitude of $\overrightarrow{M h}$, i.e., $\|\overrightarrow{M h}\|^{2}$, is less than a threshold $\epsilon$. The number of elements in vector $\overrightarrow{M h}$ can be 5 if dealing with color images, i.e., the change of $x$ coordinate $(\Delta x)$, the change of $y$ coordinate $(\Delta y)$, and the changes of intensities in $\mathrm{RGB}(\Delta R, \Delta G, \Delta B)$. For grayscale image, only three elements are needed for $\overrightarrow{M h}$, i.e., $\Delta x, \Delta y$, and $\Delta I$. If we use $S$ to denote the window centered at $P_{a}$, and use $s$ to denote a pixel within the window, $\Delta x$ can be calculated as (1), in which $I_{s}, x_{s}$, and $w_{s}$ are the intensity, the $x$ coordinate, and the weight of pixel $P_{s}$, respectively. The weight of each pixel in an image is specified by a predefined weight map.

$$
\Delta x=\sum_{s \in S} K\left(\frac{I_{s}-I_{a}}{h_{r}}\right) w_{s} x_{s}, \text { where } K(x)= \begin{cases}1 & \text { if }\|x\|<1 \\ 0 & \text { if }\|x\| \geq 1\end{cases}
$$

$\Delta y$ and $\Delta I$ are computed in a similar way. A new center of the window $P_{b}$ is calculated as $\overrightarrow{P_{b}} \leftarrow \overrightarrow{P_{a}}+\overrightarrow{M h}$. Then the mean shift vector to $P_{b}$ is calculated until the center of the window no longer shifts.

The above computation in the mean shift filtering on a pixel is implemented in a GPU kernel function. When the kernel is launched, each pixel in the source image is handled by a GPU thread. If the size of image is $m \times n, m \times n$ threads are created and scheduled to execute on hundreds of processing cores on a GPU device. 


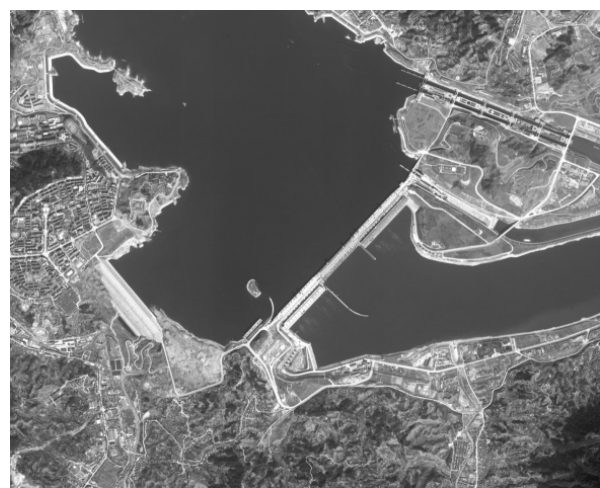

(a) Original image $(1,164 \times 945)$.

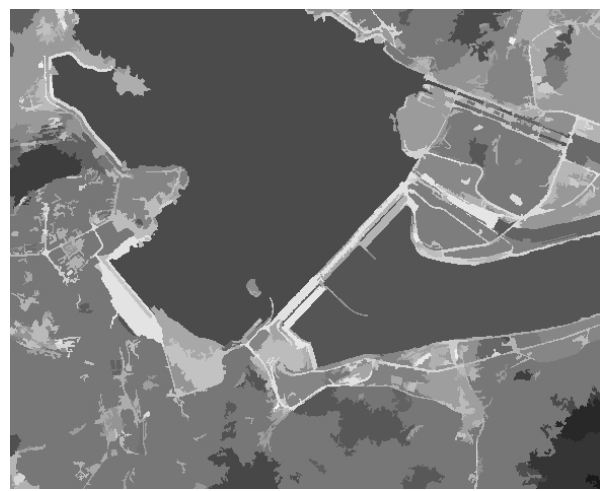

(c) Segmented image.

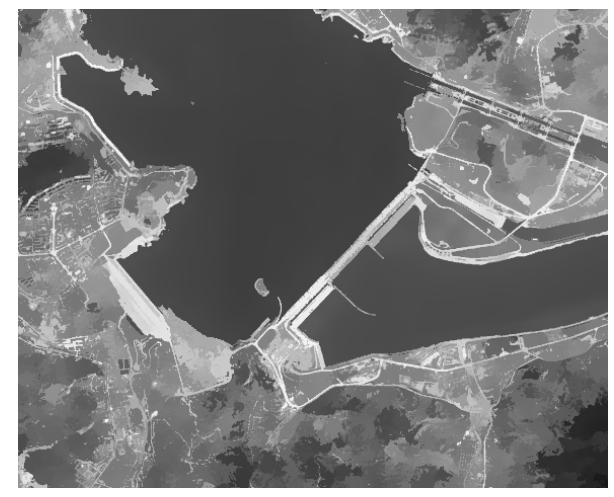

(b) Filtered image.

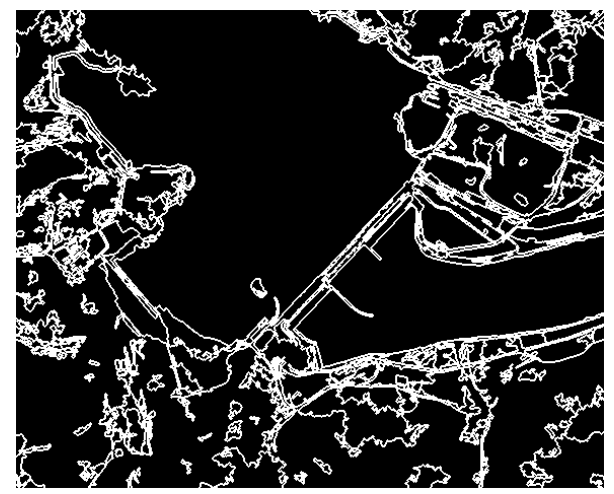

(d) Region boundaries.

Fig. 3. Image segmentation on an airborne image using mean shift.

\section{Experiments and Results}

The platform is a hybrid workstation consisting of an Intel Core i7-930 quadcore CPU and a Tesla C2075 GPU. The system has a main memory of 18 GB and runs Ubuntu 10.04.2. Tesla C2075 is based on Nvidia's Fermi GPU architecture, which is shown in Fig. 2. Fermi architecture consists of 16 streaming multiprocessors (SMs), each of which contains 32 cores. On Tesla C2075, only 14 SMs are available, providing a total 448 cores. There are total 6 GB on-board global memory available, providing a $144 \mathrm{~GB} / \mathrm{sec}$ bandwidth. The $768 \mathrm{~KB}$ L2 cache and the $64 \mathrm{~KB}$ shared memory/L1 cache further provide a good memory hierarchy for exploring data locality. 


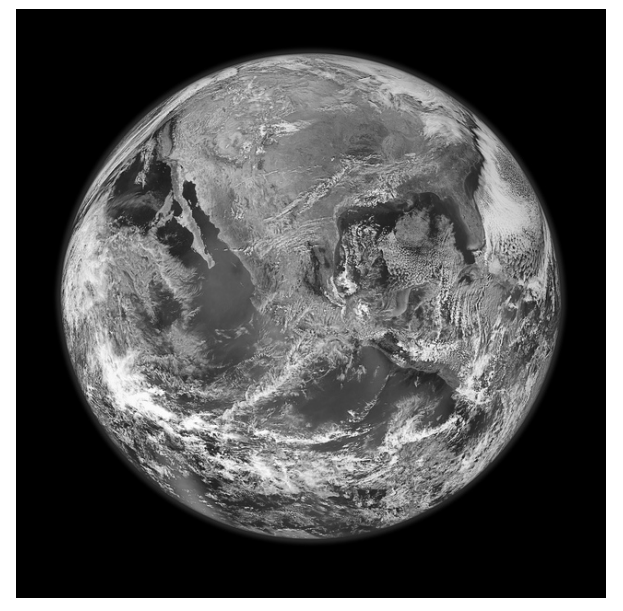

(a) Original image $(640 \times 640)$.

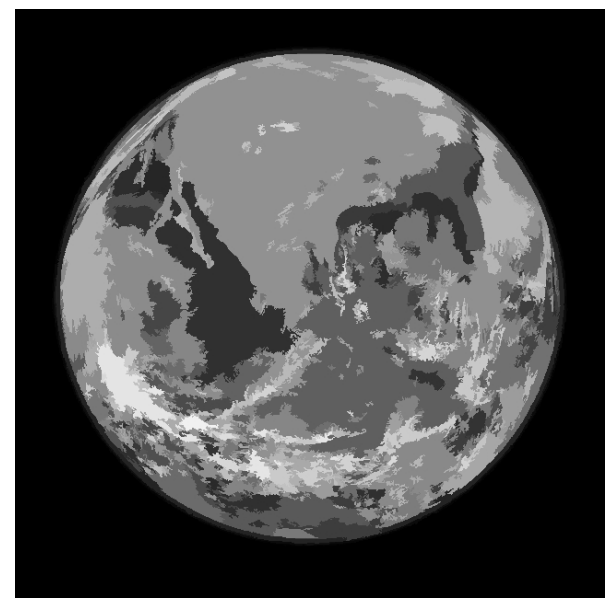

(b) Segmented image.

Fig. 4. Image segmentation on a spaceborne image using mean shift.

The GPU implementation of the mean shift filtering process contains only one GPU kernel function, which is responsible for generating one pixel in the filtered image. Therefore the number of GPU threads is same to the number of pixels in the original image. These threads are grouped into 1-dimensional thread blocks, each of which contains 128 threads. Due to the improved memory hierarchy on Fermi GPU architecture, typical performance optimization techniques, such as memory coalescing and memory prefetching [8], are not implemented in the mean shift filtering.

We first applied both versions of the mean shift segmentation algorithm on the image shown in Fig. 3(a), which is an airborne image of a water dam. Fig. 3(b) shows the image after applying mean shift filtering. It can be seen that the filtered image has been segmented into numerous regions with unnecessary details. For example, different parts of a hill may belong to different regions. After applying the region fusion step, which is carried on CPU, many neighbor regions are fused together, providing a more meaningful segmentation as shown in Fig. 3(c). The corresponding boundaries between the regions are shown in Fig. 3(d). For this airborne image, the software implementation of the mean shift filtering process takes $176.24 \mathrm{~s}$, while the GPU implementation takes only $11.44 \mathrm{~s}$, achieving a $15 \times$ speedup.

In order to further demonstrate the benefit of the hybrid implementation, we tested both implementations on spaceborne globe images of various resolutions. The globe image with resolution $640 \times 640$ and its segmentation are shown in Fig. 4. The clouds are difficult to be distinguished from the oceans and the lands in grayscale images. However, as shown in Fig. 4(b), mean shift algorithm 
Table 1. Performance improvement on mean shifter filtering.

\begin{tabular}{c|c|c|c||c|c|c|c}
\hline \multirow{2}{*}{$\begin{array}{c}\text { Image } \\
\text { Size }\end{array}$} & \multicolumn{3}{|c||}{ Processing Time $(s)$} & \multirow{2}{*}{$\begin{array}{c}\text { Image } \\
\text { Size }\end{array}$} & \multicolumn{2}{c|}{ Processing Time $(s)$} \\
\cline { 8 - 10 } \cline { 6 - 8 } & CPU & GPU & Speedup & GPU & Speedup \\
\hline $240 \times 240$ & 4.67 & 0.42 & 11.12 & $1,024 \times 1,024$ & 86.70 & 5.35 & 16.21 \\
\hline $320 \times 320$ & 8.25 & 0.62 & 13.31 & $2,048 \times 2,048$ & 354.91 & 18.01 & 19.71 \\
\hline $500 \times 500$ & 19.06 & 1.37 & 13.91 & $4,096 \times 4,096$ & 1321.76 & 61.59 & 21.46 \\
\hline $640 \times 640$ & 31.26 & 2.19 & 14.27 & $8,000 \times 8,000$ & 4046.15 & 202.5 & 19.98 \\
\hline
\end{tabular}

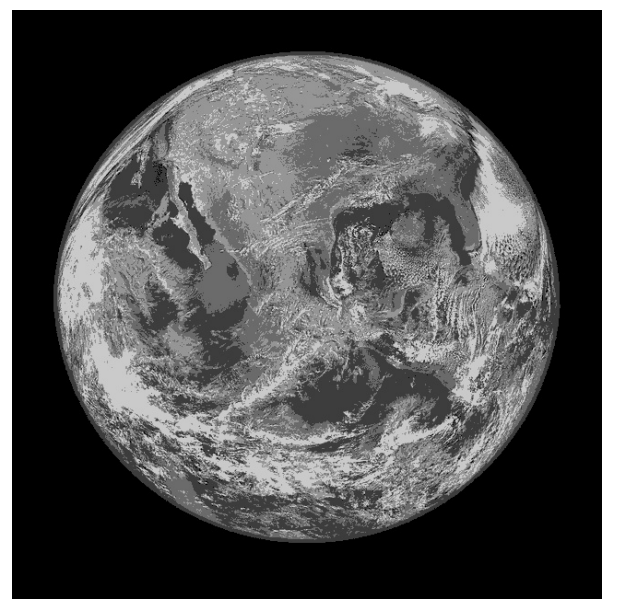

(a) $k=5$.

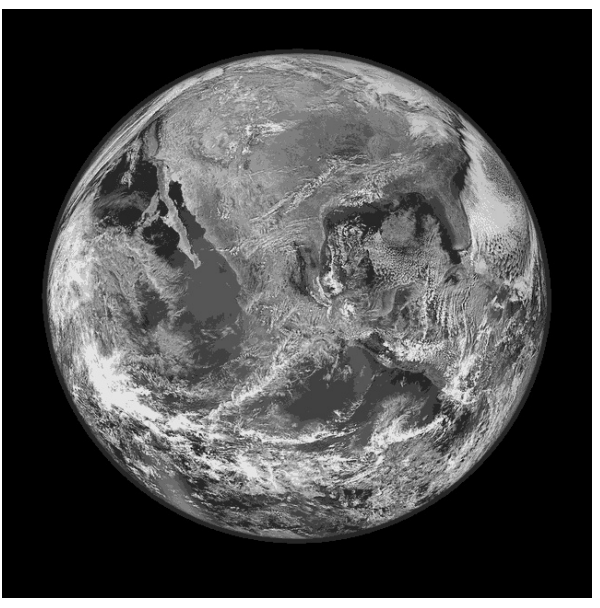

(b) $k=10$.

Fig. 5. Segmentation using $k$-means.

is capable of providing a meaningful segmentation by separating lands, oceans, and clouds. Since the region fusion step is currently carried on CPU in both pure software implementation and hybrid implementation, only the performance of mean shift filtering is shown in Tabel 1. It can be found that the performance improvement climbs as the size of image increases until hitting a plateau around $20 \times$. When the image resolution increases, more GPU thread blocks are available to be scheduled to SMs, resulting in a higher GPU occupancy and a better performance. Once the occupancy is maximized, adding more thread blocks cannot further increase the processing throughput.

It is noticed that the time spent on the region fusion step is negligible compared with the mean shift filtering step when the image size is smaller than $2,048 \times 2,048$. However, the complexity of the region fusion increases exponentially when the image becomes larger, i.e., $>2,048 \times 2,048$. Therefore one of our future work is to accelerate the region fusion step on GPU to deal with large images. 
One other very popular image segmentation algorithm is $k$-means, which is extremely faster compared with mean shift algorithm. The $k$-means algorithm is an iterative technique that is used to partition an image into $k$ clusters. The basic algorithm is as follows.

1. Pick $k$ cluster centers, either randomly or based on some heuristic;

2. Assign each pixel in the image to the cluster that minimizes the distance between the pixel and the cluster center;

3. Re-compute the cluster centers by averaging all of the pixels in the cluster;

4. Repeat steps 2 and 3 until convergence is reached (e.g., no pixels change clusters).

The quality of the solution depends on the initial set of clusters and the value of $k$. The most common form of the algorithm uses an iterative refinement heuristic known as Lloyd's algorithm [6]. Lloyd's algorithm starts by partitioning the input points into $k$ initial sets, either at random or using some heuristic data. It then calculates the mean point, or centroid, of each set. It constructs a new partition by associating each point with the closest centroid. Then the centroids are recalculated for the new clusters, and algorithm repeated by alternate application of these two steps until convergence, which is obtained when the points no longer switch clusters (or alternatively centroids are no longer changed). We implemented the Lloyd's algorithm and applied on the globe image as the results shown in Fig. 5 . It can be seen that $k$-means algorithm does not produce quite meaningful segmentation when dealing with complex remote sensing images.

\section{Conclusions}

Mean shift is a robust and non-parametric image segmentation algorithm that is capable of generating better results than other algorithms such as $k$-means. However, it is a computationally intensive process taking hours to deal with large images. Thanks to the intrinsic parallelism of mean shift algorithm, GPU can be leveraged to accelerate its performance. In this work, the mean shift filtering step is implemented on GPU. The experimental results on a Tesla C2075 GPU demonstrate a $20 \times$ speedup compared with an Intel i7-930 CPU. Future work includes (1) applying performance optimization techniques to mean shift filtering step, and (2) accelerating region fusion step on GPUs.

\section{References}

1. Cheng, Y.: Mean shift, mode seeking, and clustering. IEEE Trans. Pattern Anal. Machine Intell. 17(8), 790-799 (Aug 1995)

2. Christoudias, C.M., Georgescu, B., Meer, P.: Synergism in low level vision. In: Proc. 16th International Conference on Pattern Recognition (ICPR'02). pp. 150-155 (Aug 2002)

3. Comaniciu, D., Meer, P.: Mean shift: A robust approach towards feature space analysis. IEEE Trans. Pattern Anal. Machine Intell. 24(5), 603-619 (May 2002) 
4. Fukunaga, K., Hostetler, L.D.: The estimation of the gradient of a density function, with applications in pattern recognition. IEEE Trans. Inform. Theory 21(1), 32-40 (Jan 1975)

5. Kanungo, T., Mount, D.M., Netanyahu, N.S., Piatko, C.D., Silverman, R., Wu, A.Y.: An efficient k-means clustering algorithm: Analysis and implementation. IEEE Trans. Pattern Anal. Machine Intell. 24(5), 881-892 (Jul 2002)

6. Lloyd, S.P.: Least squares quantization in PCM. IEEE Trans. Inform. Theory 28(2), 129-137 (Mar 1982)

7. Shapiro, L.G., Stockman, G.C.: Computer Vision. Prentice Hall, Upper Saddle River, New Jersey (Feb 2001)

8. Stratton, J., Anssari, N., Rodrigues, C., Sung, I.J., Obeid, N., Chang, L., Liu, G.D., mei Hwu, W.: Optimization and architecture effects on GPU computing workload performance. In: Proc. 2012 Innovative Parallel Computing: Foundations \& Applications of GPU, Manycore, and Heterogeneous Systems (InPar2012). pp. 1-10 (May 2012) 